\title{
Faint super-soft X-ray sources in XMM-Newton Large Magellanic Cloud fields
}

\author{
P. Kahabka ${ }^{1}$, F. Haberl ${ }^{1}$, M. Pakull ${ }^{2}$, W. C. Millar ${ }^{3}$, G. L. White ${ }^{3}$, M. D. Filipović ${ }^{4}$, and J. L. Payne ${ }^{3}$ \\ 1 Max-Planck-Institut für extraterrestrische Physik, Giessenbachstrasse, 85741 Garching, Germany \\ e-mail: [pkahabka; fwh] @mpe.mpg.de \\ 2 Observatoire de Strasbourg, 11 rue de l'Université, 67000 Strasbourg, France \\ e-mail: pakull@astro.u-strasbg.fr \\ 3 Centre for Astronomy, James Cook University, Townsville, Queensland 4811, Australia \\ ${ }^{4}$ University of Western Sydney, Locked Bag 1797, Penrith South DC, NSW 1797, Australia
}

Received 23 August 2007 / Accepted 16 December 2007

\section{ABSTRACT}

\begin{abstract}
Aims. We report the discovery of three faint, super-soft X-ray sources in LMC fields observed with XMM-Newton. Methods. We analyse the three new sources together with RX J0537.7-7034 and RX J0507.1-6743, both known since ROSAT. Results. We identify XMMU J050803.1-684017 with the LMC planetary nebula LHA 120-N 102 = LMC SMP 29. The EPIC-pn spectrum of XMMU J050803.1-684017 can be fitted with a blackbody spectrum with an effective temperature of $\sim(26-51) \mathrm{eV}$ and a bolometric luminosity of $(0.1-30) \times 10^{36} \mathrm{erg} \mathrm{s}^{-1}$, depending on the assumed absorption. The source is consistent with the nucleus of a planetary nebula. The EPIC-pn spectrum of XMMU J052530.5-671501 is characterized by a blackbody temperature of $\sim(38-120) \mathrm{eV}$, a best-fit bolometric luminosity of $\sim 3 \times 10^{37} \mathrm{erg} \mathrm{s}^{-1}$ and absorbing LMC column of $\sim 8_{-8}^{+2} \times 10^{21} \mathrm{~cm}^{-2}$. This most likely highlyabsorbed super-soft source resembles the LMC super-soft source CAL 87. The EPIC-pn spectrum of XMMU J052215.0-701658 yields a blackbody temperature of $\sim(24-83) \mathrm{eV}$, but there are only about 40 source counts and the spectral parameters of the source are not well constrained. We observe RX J0537.7-7034 with EPIC-pn to be about a factor of 15 fainter compared to the ROSAT observation 11 years earlier. RX J0507.1-6743 is observed as an absorbed super-soft source by XMM-Newton and the improved $\mathrm{X}$-ray position coincides with a MACHO star which is consistent with a symbiotic star.
\end{abstract}

Key words. galaxies: Magellanic Clouds - X-rays: stars - stars: binaries: close - stars - binaries: general - stars: general

\section{Introduction}

Super-soft X-ray sources are luminous, $\sim 10^{36}-10^{38} \mathrm{erg} \mathrm{s}^{-1}$, and have soft, $k T \sim 20-80 \mathrm{eV}$, spectra (e.g. Kahabka \& van den Heuvel 1997). They have been discovered in the direction of more than 10 external galaxies (Kahabka 2006; Pietsch et al. 2005; DiStefano et al. 2006). Eight super-soft sources that were optically identified and for which an orbital period was determined are known in the Magellanic Clouds. Single-star supersoft sources like nuclei of planetary nebulae and post-AGB stars (PG1159 stars) are also known (see discussion in Kahabka \& van den Heuvel 1997). Cooling neutron stars (NSs) can have very soft spectra but with much lower luminosities. Also, magnetic cataclysmic variables ( $\mathrm{CVs}$, polars) have a super-soft spectral component. These low-luminosity sources can only be detected if they are nearby (Galactic) (see discussion in Kahabka \& Haberl 2006). Binary super-soft sources with luminosities of $\sim 10^{36}-10^{38} \mathrm{erg} \mathrm{s}^{-1}$ can be explained by steady or stable nuclear burning white dwarfs (WDs) accreting H-rich matter from a (slightly evolved) main-sequence star, subgiant, or giant (van den Heuvel et al. 1992). The number of such systems in the Galaxy can be predicted from population synthesis calculations, but they are difficult to detect due to absorption by gas in the Galactic plane (Rappaport et al. 1994). The Magellanic Clouds are better suited to detect and study super-soft sources due to lower metallicities and lower mean absorbing columns.

We used XMM-Newton archival observations to search for new super-soft sources in Magellanic Cloud fields. The $\mathrm{X}$-ray and optical investigation of one new super-soft source,
XMMU J052016.0-692505, which is a likely WD Be/X-ray binary in the LMC was reported by Kahabka et al. (2006). Three ROSAT-discovered, super-soft sources in the field of the Small Magellanic Cloud (SMC) were observed with XMM-Newton (Kahabka \& Haberl 2006). One of them is identified with the symbiotic LIN 358, a second source can be a Galactic CV, quiescent low-mass X-ray binary, or isolated cooling NS. A third source could not be detected with XMM-Newton. Here we report about three new super-soft sources discovered in archival XMM-Newton observations of the LMC field. In addition, we investigate XMM-Newton observations of the ROSATdiscovered, super-soft source RX J0537.7-7034 and the candidate RX J0507.1-6743 in the LMC.

\section{Search for super-soft sources in archival XMM-Newton LMC fields}

We investigated the EPIC-pn observations from the public $X M M-N e w t o n$ archive in the direction toward the LMC, which were performed in large-window, full-frame, or extended fullframe imaging mode (a total of more than 50 observations). We applied a maximum likelihood source detection analysis to the data in the four energy bands $0.2-0.5 \mathrm{keV}$ (with count rate $\mathrm{S}$ in this band), $0.5-1.0 \mathrm{keV}(\mathrm{H}), 1.0-2.0 \mathrm{keV}(\mathrm{H} 1)$, and $2.0-4.5 \mathrm{keV}$ (H2) and investigated the detected sources in the hardness ratio $\mathrm{HR} 1-\mathrm{HR} 2$ plane (with HR1 $=(\mathrm{H}-\mathrm{S}) /(\mathrm{H}+\mathrm{S}))$ and HR2 = $(\mathrm{H} 1-\mathrm{H})) /(\mathrm{H} 1+\mathrm{H}))$. A source that fulfilled the hardness ratio criterion $-1.0 \leq \mathrm{HR} 1 \leq 0.0$ and $-1.0 \leq \mathrm{HR} 2 \leq-0.5$ was checked on a displayed image. If confirmed as a real source and 
Table 1. Summary of XMM-Newton observations.

\begin{tabular}{lccccc}
\hline \hline Target & $\begin{array}{c}\text { EPIC-pn } \\
\text { Filter }\end{array}$ & Sat. Revol. / Obs.-ID & Observation & $\begin{array}{c}\text { Exp. } \\
{[\mathrm{ks}]}\end{array}$ \\
\hline N103B & Medium & $106 / 0113000301$ & July 07 2000, 08:01 & July 07 2000, 11:18 & 10.6 \\
N51D & Thin & $347 / 0071940101$ & Oct. 31 2001, 20:50 & Nov. 01 2001, 04:38 & 24.6 \\
N LMC 2000 (a) & Thin & $115 / 0127720201$ & July 25 2000, 22:48 & July 26 2000, 03:23 & 14.6 \\
N LMC 2000 (b) & Thin & $132 / 0127720301$ & Aug. 28 2000, 13:53 & Aug. 28 2000, 16:43 & 10.2 \\
RX J0537.7-7034 & Thin & $673 / 0148870501$ & Aug. 12 2003, 08:36 & Aug. 12 2003, 23:02 & 37.8 \\
DEM L71 (a) & Medium & $241 / 0109990201$ & Apr. 02 2001, 12:32 & Apr. 03 2001, 00:30 & 25.7 \\
DEM L71 (b) & Medium & $735 / 0201840101$ & Dec. 13 2003, 20:39 & Dec. 14 2003, 13:25 & 52.9 \\
\hline
\end{tabular}

not as an artifact (e.g. a spurious detection in the halo of a bright source) then an image was binned in detector coordinates. If the source counts were recorded in one single pixel or in a $4 \times 4$ matrix or a similar matrix then we rejected the source as a hot pixel or as caused by a particle event.

In total, we discovered four new super-soft sources that we further investigated by cross-correlating with optical catalogs (using Vizier). Three of the super-soft sources are presented and discussed here, the fourth super-soft source is described in Kahabka et al. (2006). We could not confirm the super-soft source XMMU J053056.2-6548009 (Haberl et al. 2003), which turns out to be a detector artifact. We also include in our sample the ROSAT detected super-soft sources RX J0537.7-7034 and RX J0507.1-6743, located in the fields of XMM-Newton observations. RX J0507.1-6743 is observed with HR1 > 0.0, in agreement with an absorbed super-soft source. If a sufficient number of source counts $\gtrsim 50$ were collected then an X-ray spectral fit (using an absorbed blackbody model) was applied to the data and the spectral parameters were derived. The observations, which include the sources discussed here, are summarized in Table 1. During all seven observations, EPIC-pn was operated in full-frame read-out mode with $73 \mathrm{~ms}$ frame time.

In Table 2, we give the source existence likelihood ratio (ML) for the soft $(0.2-0.5 \mathrm{keV})$ and the hard $(0.5-1.0 \mathrm{keV})$ spectral band, the equatorial source coordinates (RA and Dec) with $68 \%$ confidence error radius, vignetting corrected source count rates in the soft and hard band, and hardness ratios HR1 and HR2, when available. We observed XMMU J052215.0-701658 and RX J0507.1-6743 twice, marked as (a) and (b) in Table 1.

\subsection{EPIC data}

We used data collected with the European Photon Imaging Cameras (EPIC) with the $p n$ and two MOS CCD detectors (Strüder et al. 2001; Turner et al. 2001) located behind the X-ray telescopes (Aschenbach et al. 2000). Spectral analysis was performed from $E P I C$ - $p n$ data only.

We created EPIC-pn event files with the tool "epchain" from the XMM-Newton analysis package (SAS 7.0) using the parameter setting screenlowthresh $=0$. In the extraction of the spectrum, we used single and double pixel events. For spectral fitting, we calculated effective areas and redistribution matrices for $E P I C$ $p n$ using "arfgen" and "rmfgen". We accumulated source spectra with a minimum of $20,35,15,30$, and 50 counts per spectral bin for each of the five sources listed in Table 3.

We performed spectral fits using XSPEC v11.3.2 based on C-statistics (Cash 1979) since there were generally less than 10 background subtracted source counts per spectral bin. We used a simple blackbody model (the spectral resolution of the instrument at lowest energies is insufficient for more sophisticated models) modified by Galactic absorption with the solar elemental abundances of Anders \& Grevesse (1989) and absorption due to LMC gas. The results ${ }^{1}$ are summarized in Table 3 , which includes absorbing column density due to LMC gas $N_{\mathrm{H}}^{\mathrm{LMC}}$, blackbody temperature $k T_{\mathrm{bb}}$, observed and absorption corrected flux, and intrinsic source luminosity for a distance of $50 \mathrm{kpc}$ in the $0.1-2.4 \mathrm{keV}$ energy band. Errors denote $90 \%$ confidence limits. The C-statistic (C-stat) and the number of spectral bins (Nbin) are listed in the final columns. Also given are the absorption column densities due to Galactic gas (fixed in the fit) and the total LMC gas as determined from $21-\mathrm{cm}$ measurements in the direction of the source (Brüns et al. 2005), for comparison with the value derived from the fit.

For J050803, we extracted the source counts in a circle with radius $15^{\prime \prime}$ and the background counts from a nearby circle with radius $15^{\prime \prime}$ and collected $\sim 122$ source counts for X-ray spectral fitting. In the case of J052530, we extracted the source counts in a circle with radius $35^{\prime \prime}$ and the background counts from a nearby circle with radius $35^{\prime \prime}$ and collected 60 source counts for X-ray spectral fitting. J052215 was discovered in an archival observation of the LMC classical nova N LMC 2000 performed on 25 July 2000. The source was also in the field of view of a second observation of N LMC 2000 performed on 28 Aug. 2000. It was only marginally detected during the second observation (cf. Table 2), but the vignetting corrected count rates of both observations are consistent within $68 \%$ confidence. We extracted the source counts in a circle with radius $15^{\prime \prime}$ and the background counts from a nearby circle with radius $15^{\prime \prime}$ and collected 32 counts for X-ray spectral fitting. In the analysis of J0537, we obtain $\sim 64$ and for J0507, $\sim 138$ source counts for X-ray spectral fitting.

In Fig. 1, we present the EPIC-pn spectra of the investigated sources together with their best-fit models. Figure 2 shows the confidence contours for the spectral parameters $N_{\mathrm{H}}^{\mathrm{LMC}}$ and $k T_{\mathrm{bb}}$.

The sources J050803, J0537 and J0507 are also contained in the field of view of the EPIC-MOS detectors. All three supersoft sources were detected with the MOS detectors. J052530 and J052215 were observed far off-axis and, unfortunately, were not in the field of view of the EPIC-MOS detectors. The lower efficiency of the $M O S$, in particular at low energies, does not yield a sufficient number of counts for a spectral analysis.

We generated the EPIC-pn light curves of the five super-soft $\mathrm{X}$-ray sources using a binsize of $3600 \mathrm{~s}(1 \mathrm{~h})$ in a "soft" $(0.12-$ $0.4 \mathrm{keV})$ or $(0.17-0.4 \mathrm{keV})$ and "hard" $(0.4-0.7 \mathrm{keV})$ band together with the hardness ratio as a function of time. Due to the small number of counts detected from these sources, we could

\footnotetext{
1 Hereafter, we abbreviate:

XMMU J050803.1-684017 = J050803,

XMMU J052530.5-671501 = J052530,

XMMU J052215.0-701658 = J052215,

RX J0537.7-7034 = J0537 and RX J0507.1-6743 = J0507.
} 
Table 2. Maximum likelihood source detection results for the investigated super-soft sources.

\begin{tabular}{|c|c|c|c|c|c|c|}
\hline \multirow[t]{2}{*}{ Source } & \multirow{2}{*}{$\begin{array}{l}\text { ML } \\
\text { soft } \\
\text { hard }\end{array}$} & \multicolumn{3}{|c|}{ Position } & \multirow{2}{*}{$\begin{array}{c}\text { Count rate } \\
\text { soft } \\
\text { hard } \\
\left(10^{-3} \mathrm{~s}^{-1}\right)\end{array}$} & \multirow{2}{*}{$\begin{array}{c}\text { Hardness ratio } \\
\text { HR1 } \\
\text { HR2 }\end{array}$} \\
\hline & & RA & 000) & Err & & \\
\hline XMMU J050803.1-684017 & $\begin{array}{c}71 \\
-\end{array}$ & $05^{\mathrm{h}} 08^{\mathrm{m}} 03^{\mathrm{s}} .1$ & $-68^{\circ} 40^{\prime} 17^{\prime \prime} .7$ & 1..1 & $\begin{array}{c}9.41 \pm 1.40 \\
-\end{array}$ & $-1.00 \pm 0.11$ \\
\hline XMMU J052530.5-671501 & $\begin{array}{l}63 \\
51\end{array}$ & $05^{\mathrm{h}} 25^{\mathrm{m}} 30.5^{\mathrm{s}}$ & $-67^{\circ} 15^{\prime} 01^{\prime \prime} 0$ & 1 ." 4 & $\begin{array}{l}7.46 \pm 1.22 \\
6.63 \pm 1.16\end{array}$ & $-0.06 \pm 0.12$ \\
\hline XMMU J052215.0-701658 (a) & $\begin{array}{c}47 \\
3\end{array}$ & $05^{\mathrm{h}} 22^{\mathrm{h}} 15.0^{\mathrm{s}}$ & $-70^{\circ} 16^{\prime} 58^{\prime \prime} .5$ & 1 ..7 & $\begin{array}{l}9.17 \pm 1.53 \\
2.30 \pm 1.08\end{array}$ & $-0.60 \pm 0.16$ \\
\hline XMMU J052215.0-701658 (b) & $\begin{array}{l}8 \\
2\end{array}$ & $05^{\mathrm{h}} 22^{\mathrm{h}} 15.6^{\mathrm{s}}$ & $-70^{\circ} 16^{\prime} 57^{\prime \prime} .5$ & $2 \prime .8$ & $\begin{array}{l}5.85 \pm 1.82 \\
3.08 \pm 1.82\end{array}$ & $-0.31 \pm 0.30$ \\
\hline RX J0537.7-7034 & $\begin{array}{c}16 \\
1\end{array}$ & $05^{\mathrm{h}} 37^{\mathrm{m}} 45.0^{\mathrm{s}}$ & $-70^{\circ} 33^{\prime} 46^{\prime \prime} 4$ & 1 .'9 & $\begin{array}{l}0.96 \pm 0.23 \\
0.31 \pm 0.20\end{array}$ & $-0.51 \pm 0.26$ \\
\hline RX J0507.1-6743 (a) & $\begin{array}{l}0 \\
2\end{array}$ & $05^{\mathrm{h}} 07^{\mathrm{m}} 10.9^{\mathrm{s}}$ & $-67^{\circ} 43^{\prime} 19^{\prime} 0$ & 4..6 & $\begin{array}{c}- \\
1.99 \pm 1.04\end{array}$ & $\begin{array}{c}- \\
-1.00 \pm 0.35\end{array}$ \\
\hline RX J0507.1-6743 (b) & $\begin{array}{l}23 \\
98 \\
\end{array}$ & $05^{\mathrm{h}} 07^{\mathrm{m}} 10.7^{\mathrm{s}}$ & $-67^{\circ} 43^{\prime} 17^{\prime \prime} .0$ & 0.9 & $\begin{array}{l}1.75 \pm 0.41 \\
5.79 \pm 0.66\end{array}$ & $\begin{array}{c}0.54 \pm 0.09 \\
-0.68 \pm 0.08\end{array}$ \\
\hline
\end{tabular}

Table 3. Spectral analysis of EPIC-pn data using a blackbody model.

\begin{tabular}{|c|c|c|c|c|c|c|c|c|c|}
\hline Source & $\begin{array}{c}N_{\mathrm{H}, 21 \mathrm{~cm}}^{\mathrm{gal}} \\
\left(10^{20} \mathrm{~cm}^{-2}\right)\end{array}$ & $\begin{array}{c}N_{\mathrm{H}, 21 \mathrm{~cm}}^{\mathrm{LMC}} \\
\left(10^{20} \mathrm{~cm}^{-2}\right)\end{array}$ & $\begin{array}{c}N_{\mathrm{H}}^{\mathrm{LMC}} \\
\left(10^{20} \mathrm{~cm}^{-2}\right)\end{array}$ & $\begin{array}{l}k T_{\mathrm{bb}} \\
(\mathrm{eV})\end{array}$ & $\begin{array}{l}\text { flux }^{\text {abs }} \\
\left(10^{-13}\right.\end{array}$ & $\begin{array}{c}\text { flux }{ }^{\text {unabs }} \\
\left.\operatorname{erg} \mathrm{cm}^{-2} \mathrm{~s}^{-1}\right)\end{array}$ & $\begin{array}{c}L^{\text {unabs }} \\
\left(10^{36} \mathrm{erg} \mathrm{s}^{-1}\right)\end{array}$ & C-stat & Nbin \\
\hline J050803 & 5.2 & 14 & $2_{-2}^{+10}$ & $41_{-15}^{+10}$ & 0.27 & 6.7 & 0.20 & 8.6 & 9 \\
\hline J052530 & 4.3 & 71 & $81_{-78}^{+20}$ & $46_{-8}^{+74}$ & 0.10 & 1050 & 31 & 7.4 & 7 \\
\hline J052215 (a) & 5.0 & 7.2 & $\leq 43$ & $58_{-34}^{+25}$ & 0.13 & 0.75 & 0.022 & 2.8 & 3 \\
\hline J052215 (b) & & & & & 0.10 & 0.58 & 0.017 & & \\
\hline J0537 & 4.7 & 29 & $15_{-15}^{+30}$ & $29_{-11}^{+28}$ & 0.019 & 400 & 12 & 3.4 & 6 \\
\hline J0507 (b) & 4.4 & 11 & $180_{-180}^{+70}$ & $66_{-6}^{+224}$ & 0.091 & 1090 & 32 & 20.4 & 11 \\
\hline
\end{tabular}

not detect significant count rate or hardness ratio variations for any of the sources.

\section{MACHO observations}

\section{1. $R X J 0507.1-6743$}

The X-ray position of J0507 is 1 '.9 from a Denis star with $B=$ $17.3, R=16.4, I=14.9$, and $J=13.3$ which is at a distance of $0 .{ }^{\prime} 5$ from the $M A C H O^{2}$ star 20.4313.548. We retrieved the $\mathrm{MACHO}$ light curve with the interactive light curve browser ${ }^{3}$. In Fig. 3, we show the $B$-and $R$-band light curves together with the $B-R$ color.

An FFT analysis was applied to the $M A C H O R$ - and $B$-band data with a magnitude error $<0.05$ mag (Lomb 1976; Scargle $1982)$. We found high power $(\sim 55)$ for a period of $374_{-9}^{+10}$ days (68\% confidence) in the $B$-band data (Fig. 4, see Fig. 5 for the folded light curve), but no significant period is found in the $R$ band data. The ephemeris for the blue optical minimum is:

$\mathrm{JD}_{0}=24450154+\left(374_{-9}^{+10} \times E\right)$.

Folding the $R$-band data with the same period shows a weak modulation, but with a phase shift of $\sim 0.5$ (Fig. 5 ). As this period is close to one year, we used a control star $(B \sim 18.5) 3^{\prime \prime}$ distant from the $M A C H O$ star and generated the FFT for this control star. We found in the $B$-band data a peak of low power $(\sim 10)$ at

\footnotetext{
2 The "Massive Compact Halo Object" project.

3 www.macho.mcmaster.ca/Data/MachoData.html
}

a period of $362 \pm 9$ days, which is consistent with the one-year period.

\section{2. $X M M U$ J052530.5-671501}

J052530 has the MACHO star 60.7345 .253 as an optical counterpart at a distance of $11^{\prime \prime} 9$. In Fig. 6, we show the $B$ - and $R$-band light curve and the $B-R$ color.

From the FFT analysis of the MACHO $R$ - and $B$-band data with a magnitude error $<0.05 \mathrm{mag}$, we found no significant power for any period. For comparison, the FFT of the $B$-band data is shown in Fig. 7. Also, no significant period close to one year is found. This would be consistent with a close-binary super-soft source if the orbital period is less than $\sim 1$ day.

\section{Optical identification and spectroscopy of RX J0507.1-6743}

We obtained the optical spectrum of J0507 on 24 November 2006, using the Grating Spectrograph with SITe CCD on the $1.9 \mathrm{~m}$ (74 inch) Radcliff telescope at the South African Astronomical Observatory (SAAO) in Sutherland. The CCD is effectively $266 \times 1798$ pixels in size, with a pixel size of $15 \mu$. Using grating \#7 (300 lines $\mathrm{mm}^{-1}$ ) set at an angle of $15^{\circ}$, two spectral images between $4500 \AA$ and $7000 \AA$ with a spectral resolution of $5 \AA$ were taken.

We reduced and calibrated the spectra using NOAO IRAF software, with the instrument's $\mathrm{Cu} / \mathrm{Ar}$ arc standard and the star, 

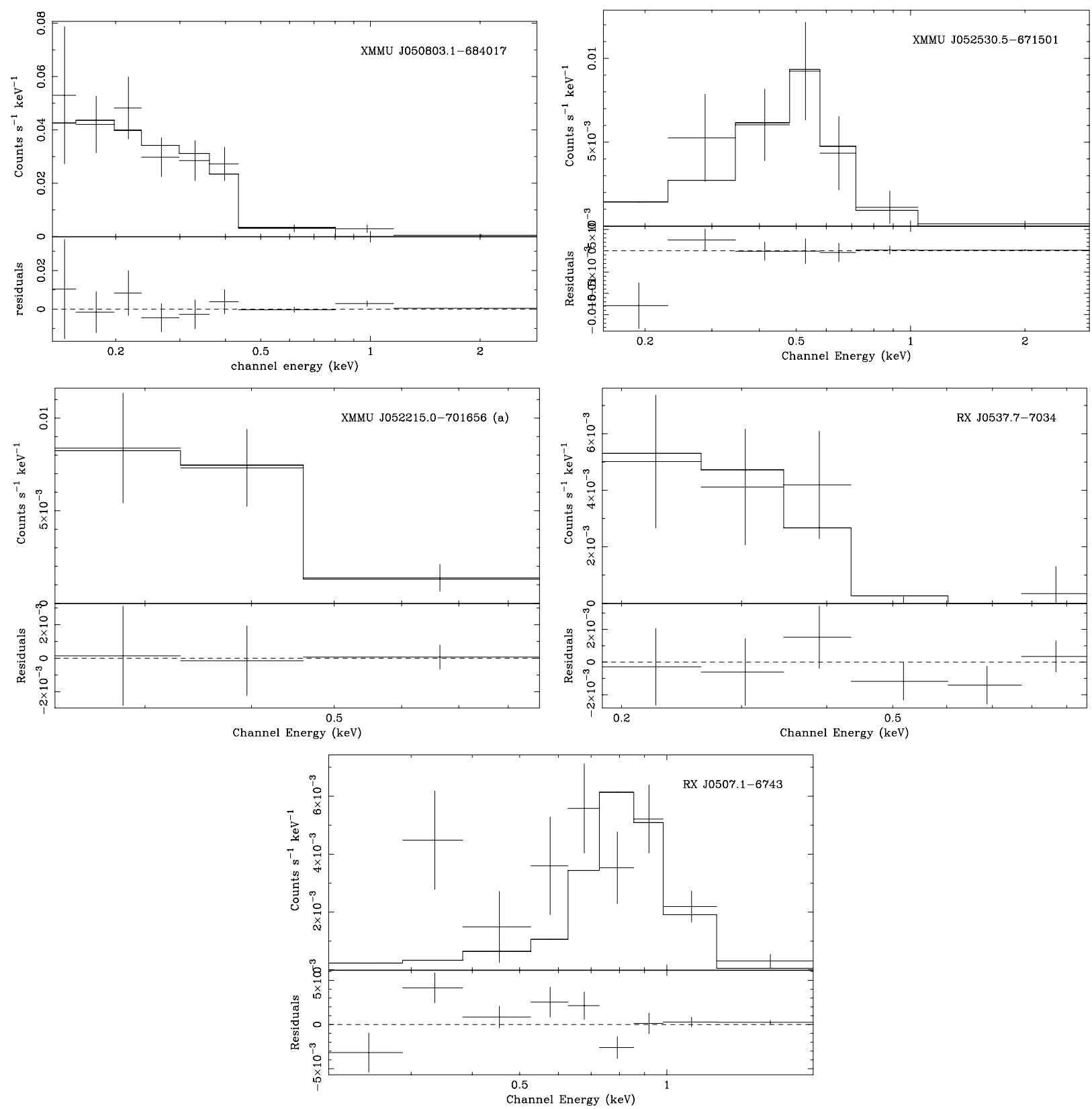

Fig. 1. EPIC-pn spectra of faint SSS with best-fit blackbody model as histogram.

Feige $110\left(\alpha=23^{\mathrm{h}} 19^{\mathrm{m}} 58.4^{\mathrm{s}}, \delta=-34^{\circ} 42^{\prime} 51^{\prime \prime}\right)$ as the flux standard. The calibrated spectrum is shown in Fig. 8.

\section{Discussion}

We report the discovery of three new faint super-soft X-ray sources in the LMC field. They have XMM-Newton EPIC-pn count rates of $\sim(1-14) \times 10^{-3} \mathrm{~s}^{-1}$. None of them were detected with ROSAT. Assuming a blackbody spectral flux distribution, the bolometric luminosity of the three sources cannot be well constrained due to the small number of observed counts. From the confidence contours of the spectral parameters (Fig. 2), it follows that the bolometric luminosity of all new sources is at least $\sim 10^{34} \mathrm{erg} \mathrm{s}^{-1}$ if they are located in the LMC.

\subsection{The planetary nebula LMC SMP 29}

J050803 is found at a distance of 2 .'7 from the position of the planetary nebula LHA 120-N 102 given in Leisy et al. (1997). Although such a distance is slightly larger than the general $X M M$-Newton position uncertainty of $\sim 2^{\prime \prime}$, the X-ray source is likely related to the planetary nebula. For the nuclei of six LMC planetary nebulae, including: LMC SMP 7 = LHA 120-N 77F, LMC SMP 29 = LHA 120-N 102, LMC SMP 32 = LHA 120N 192, LMC SMP 37 = LHA 120-N 28, LMC SMP $83=$ IRAS 05363-6720 and LMC SMP 101, effective temperatures of $(1.6-2.3) \times 10^{5} \mathrm{~K}$ and luminosities of $(1.0-1.3) \times$ $10^{37} \mathrm{erg} \mathrm{s}^{-1}$ were derived from optical and UV data. These planetary nebulae therefore are candidates for super-soft X-ray sources that should be detectable with XMM-Newton EPIC-pn. As the XMM-Newton observations covered only a few percent of the LMC so far, the chance to serendipitously cover one of these 

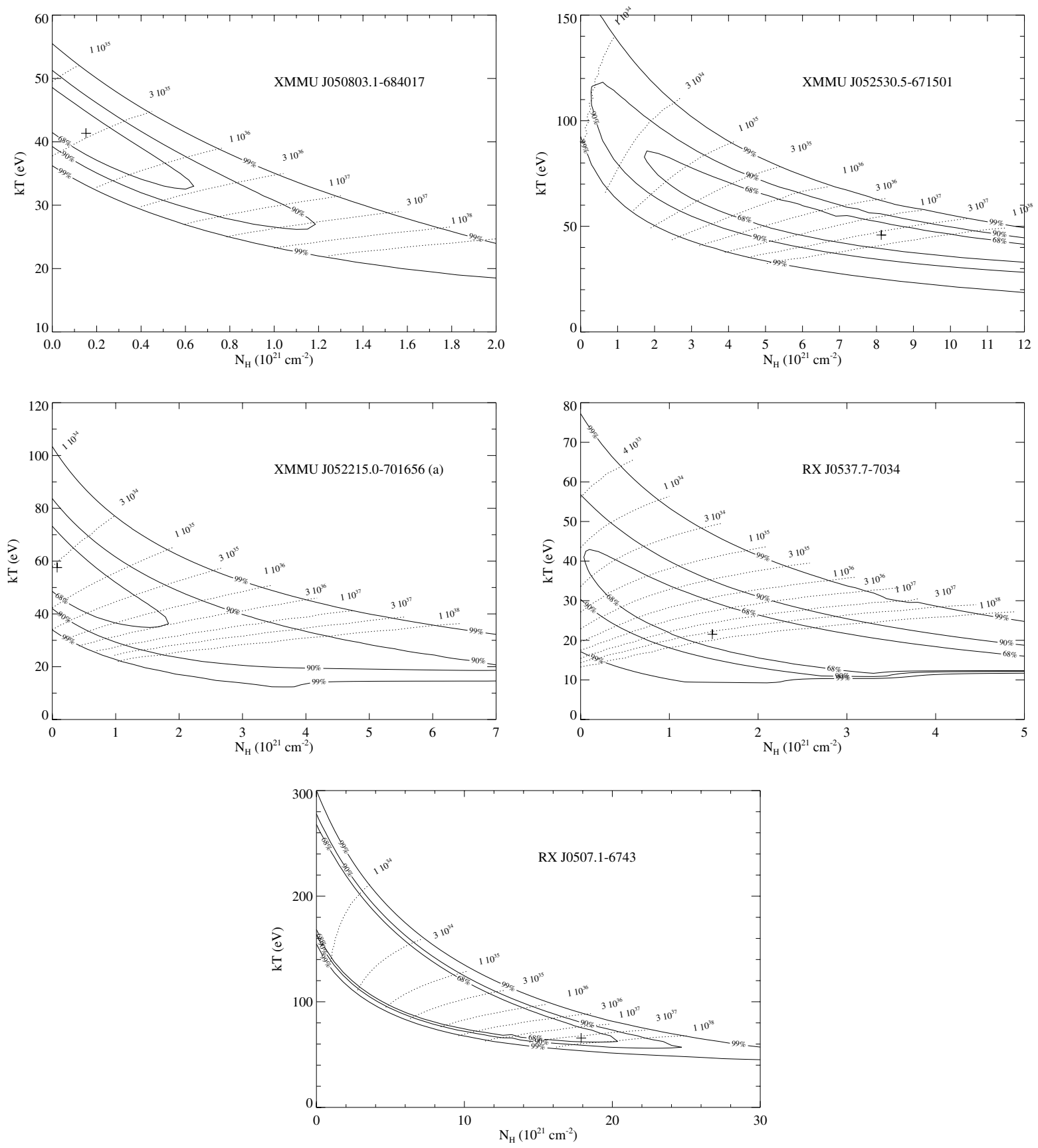

Fig. 2. $N_{\mathrm{H}}^{\mathrm{LMC}}-k T$ fit parameter plane with confidence contours. The formally best-fit results are marked with a cross and dotted lines indicate levels of constant $L_{\mathrm{bol}}\left(\right.$ in $\mathrm{erg} \mathrm{s}^{-1}$ ).

planetary nebulae is not very large. This is consistent with finding one of these LMC planetary nebulae, LMC SMP 29, as a super-soft X-ray source with XMM-Newton. The temperature and luminosity inferred from the blackbody spectral fit to the X-ray data (cf. Table 3) can be compared with the values determined from the optical and UV data. We find that the temperature inferred from the X-ray data, assuming a blackbody description of the continuum, is somewhat higher than the value inferred from the UV data.

\subsection{A highly absorbed super-soft source?}

The optical counterpart of J052530 may be an USN0-B1.0 star located 1'.8 away from the X-ray position with $B \sim 20.0$ and
$B-R \sim 1.2$. From the $\mathrm{X}$-ray spectral fit it is found that the source is probably heavily absorbed with $N_{\mathrm{H}}=8_{-8}^{+2} \times 10^{21} \mathrm{~cm}^{-2}$. The color index $B-R$ of the likely optical counterpart also suggests considerable reddening which is consistent with the absorbing column inferred from the X-ray spectrum. For comparison, the color index $B-R$ is 3.3 for the galactic super-soft source RX J0925.7-4758 (Šimon 2003), which is consistent with a large absorbing column of $\sim 10^{22} \mathrm{~cm}^{-2}$. The LMC absorbing column, inferred from the spectral fit (although quite uncertain) is consistent with the total LMC column in the direction of the source $\left(7.1 \times 10^{21} \mathrm{~cm}^{-2}\right)$ inferred from $21-\mathrm{cm}$. The unabsorbed luminosity $(0.1-2.4 \mathrm{keV})$ of $\sim 3 \times 10^{37} \mathrm{erg} \mathrm{s}^{-1}$ is consistent with the luminosity of a super-soft X-ray source. This source resembles the super-soft sources CAL 87 and RX J0925.7-4758, 


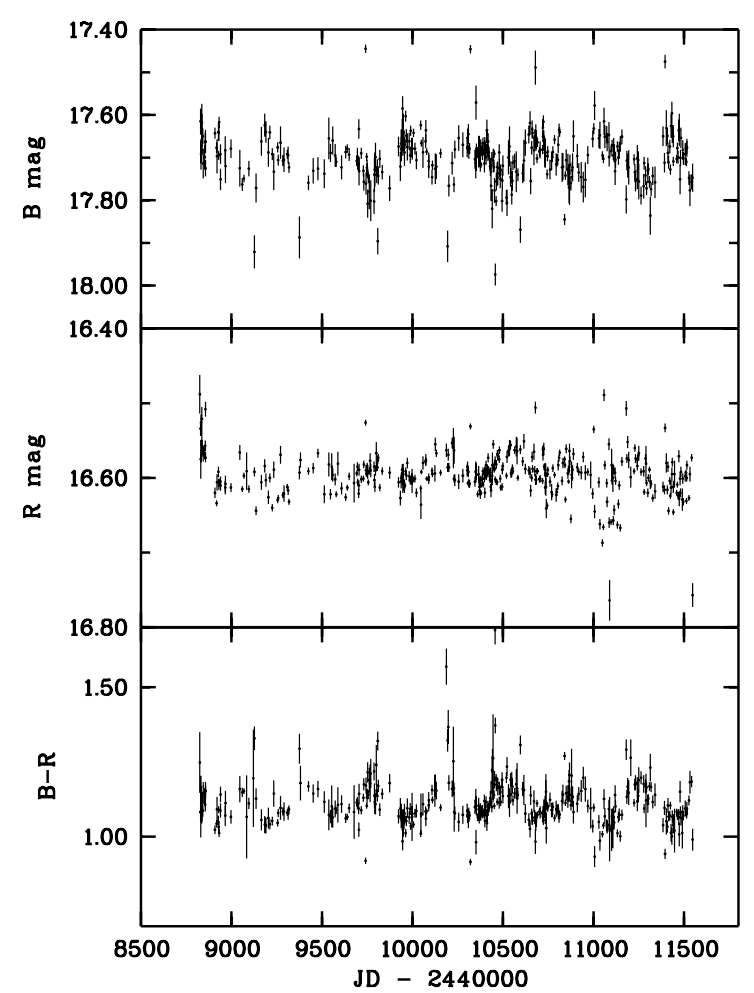

Fig. 3. $M A C H O B$ - and $R$-band light curves of RX J0507.1-6743 together with the $B-R$ color (only for data points with magnitude error $<0.05 \mathrm{mag}$ ). Uncalibrated light curves shifted in $B$ and $R$ to give a mean $B=17.7$ and $R=16.5$.

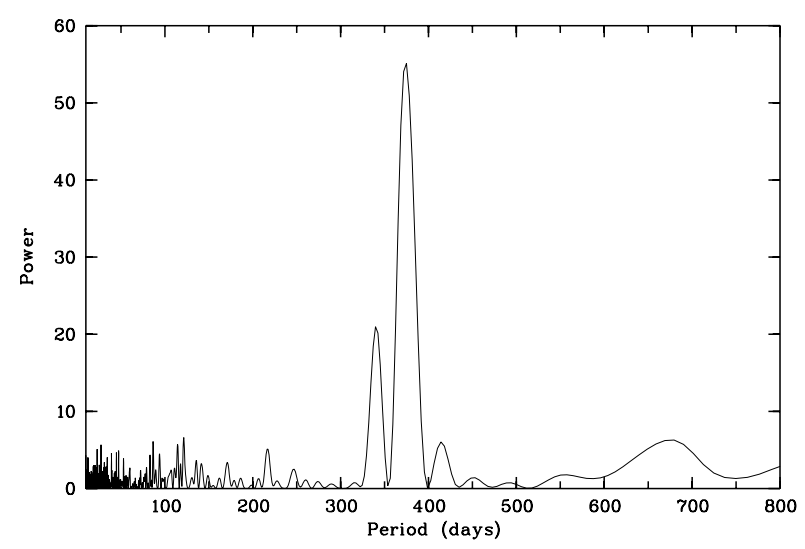

Fig. 4. FFT of the blue MACHO light curve of RX J0507.1-6743.

for which a high absorbing column has also been inferred from $\mathrm{X}$-ray spectra.

\subsection{A southern super-soft source}

No optical counterpart is found within $3^{\prime \prime}$ of the position of J052215 (from observation a) using the catalogues from Vizier. This may indicate that the optical counterpart of J052215 is optically faint. The position of the source derived from observation b deviates by $3^{\prime \prime}$ from the position of observation a, which is consistent with a $2^{\prime \prime}$ position uncertainty of the first and a $3^{\prime \prime}$ position uncertainty of the second observation. The blackbody temperature of $24-83 \mathrm{eV}$ (90\% confidence) is consistent with a super-soft X-ray source. If one constrains the absorbing column to this source to $\lesssim 7 \times 10^{20} \mathrm{~cm}^{-2}$ (the LMC column inferred from
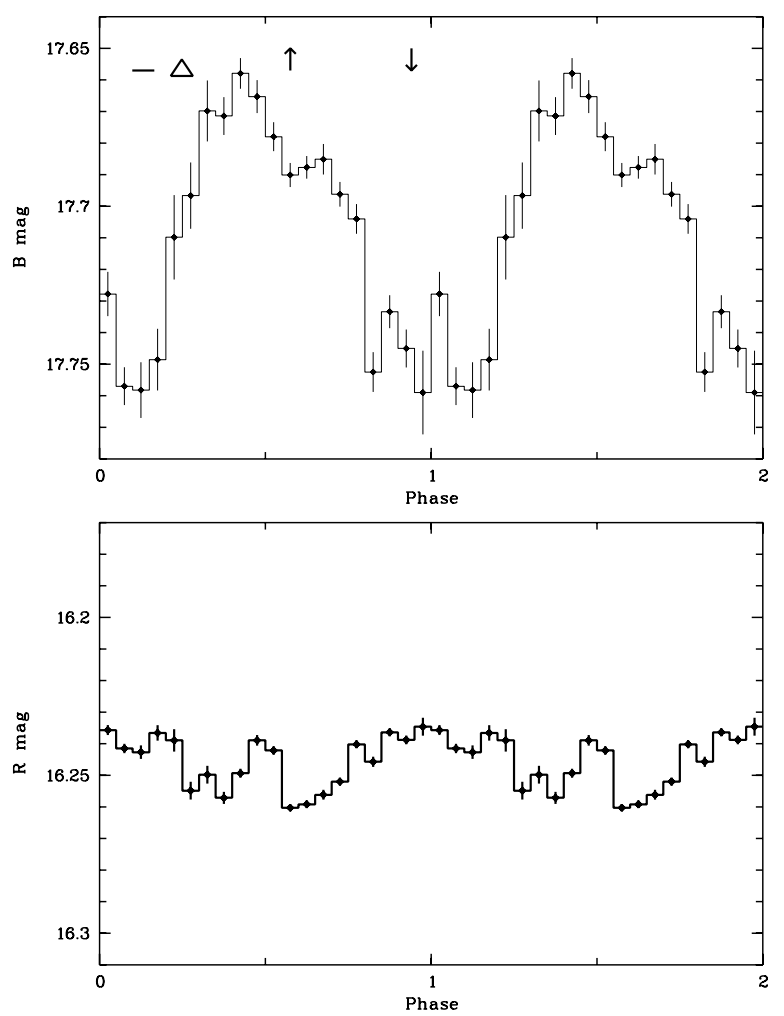

Fig. 5. Folded $B$ (upper panel) and $R$-band (lower panel) MACHO light curve of RX J0507.1-6743 for a period of 374 days and ephemeris $\mathrm{JD}_{0}=24450154$. The up and down arrows mark the phase of the XMM-Newton observations of RX J0507.1-6743 when the source was bright and faint, and the triangle and "-" the phase of the ROSAT PSPC observation when the source was bright and faint, respectively.

neutral hydrogen), then the bolometric luminosity of the source would be $\$ 3 \times 10^{35} \mathrm{erg} \mathrm{s}^{-1}$ given the distance to the LMC. But we cannot exclude a Galactic nature for the source with an even lower luminosity. The source was observed during two observations separated by one month. The count rate was consistent during both observations.

\subsection{RX J0537.7-7034, a ROSAT-discovered super-soft source}

The XMM-Newton position of J0537 is only 0.'7 distant from the optical position (Orio et al. 1997). The temperature of $29_{-11}^{+28} \mathrm{eV}$ inferred with EPIC-pn is consistent with the minimum temperature of $18 \mathrm{eV}$ and the maximum temperature of $42 \mathrm{eV}$ given by Orio et al. (1997) from the ROSAT observations. The best-fit luminosity $(0.1-2.4 \mathrm{keV})$ of $\sim 1.2 \times 10^{37} \mathrm{erg} \mathrm{s}^{-1}$ is consistent with stable nuclear burning on the surface of a WD. The observation of J0537 as a super-soft X-ray source for more than 10 years argues for a sub-class of super-soft sources with small orbital periods $\sim(3.5-4)$ hours similar to classical novae, but with considerably longer stable nuclear burning times ( $>10$ years). This argues for lower mass $\left(\sim 0.6 M_{\odot}\right)$ WDs in these systems (Greiner et al. 1994). The expected ROSAT PSPC count rate based on the EPIC-pn spectrum is a factor of 15 smaller than the PSPC count rate observed $\sim 11$ years earlier. This could indicate a decrease in luminosity and/or temperature, consistent with a lower mass WD. 


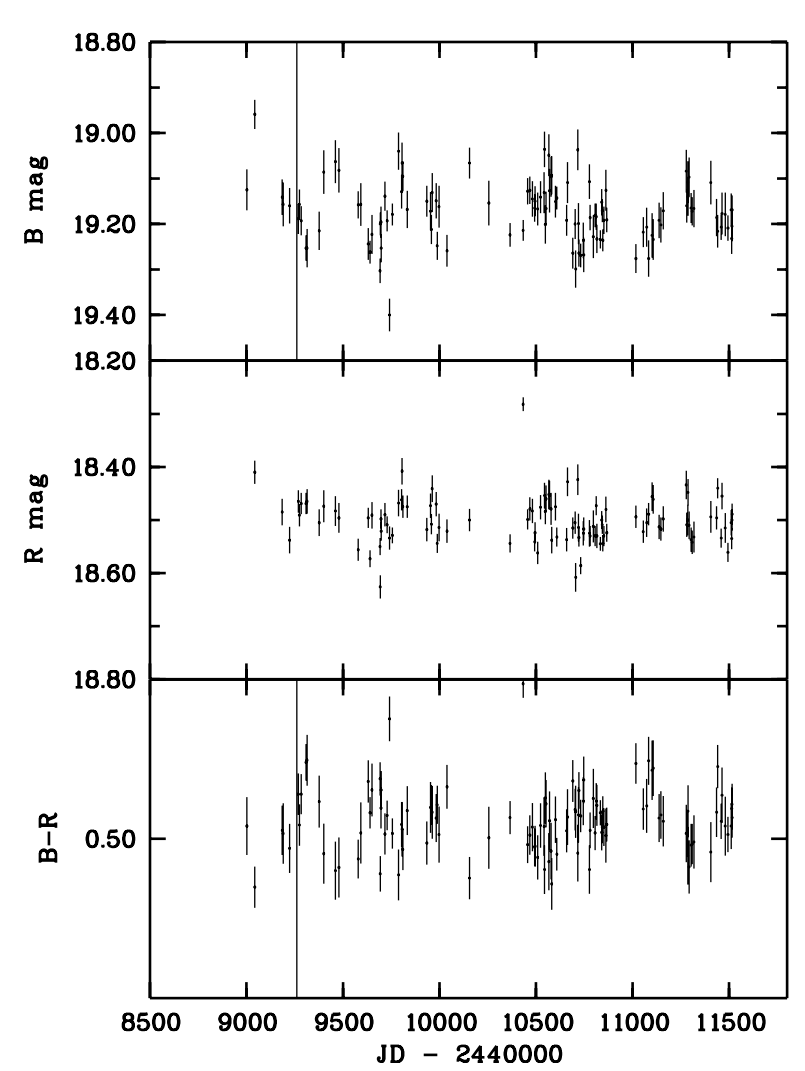

Fig. 6. MACHO $B$ and $R$ band light curve of XMMU J052530.5-671501 together with the $B-R$ color (only for data points with magnitude error $<0.05 \mathrm{mag}$ ). Uncalibrated light curves shifted in $B$ and $R$ to give a mean $B=19.2$ and $R=18.5$.

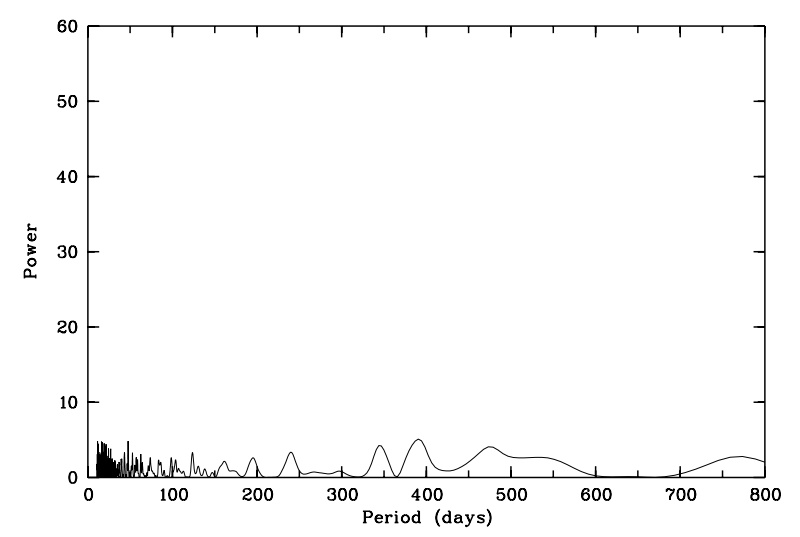

Fig. 7. FFT of the blue $M A C H O$ light curve of XMMU J052530.5-671501. The scale is the same as in Fig. 4.

\section{5. $R X$ J0507.1-6743, a variable and absorbed symbiotic?}

J0507 was covered twice by ROSAT PSPC observations separated by about one year. The source was only detected significantly during the first observation and the spectrum was very soft with counts detected below $0.4 \mathrm{keV}$. The source was also detected during a ROSAT HRI observation (Sasaki et al. 2000). Assuming the spectral model obtained from the PSPC observation, Haberl \& Pietsch (1999b) inferred a variability factor of $\sim 10-40$ and classified the source as a super-soft source in the LMC or a soft foreground CV.

J0507 was observed twice with XMM-Newton, but we detected it significantly only during the second observation. The

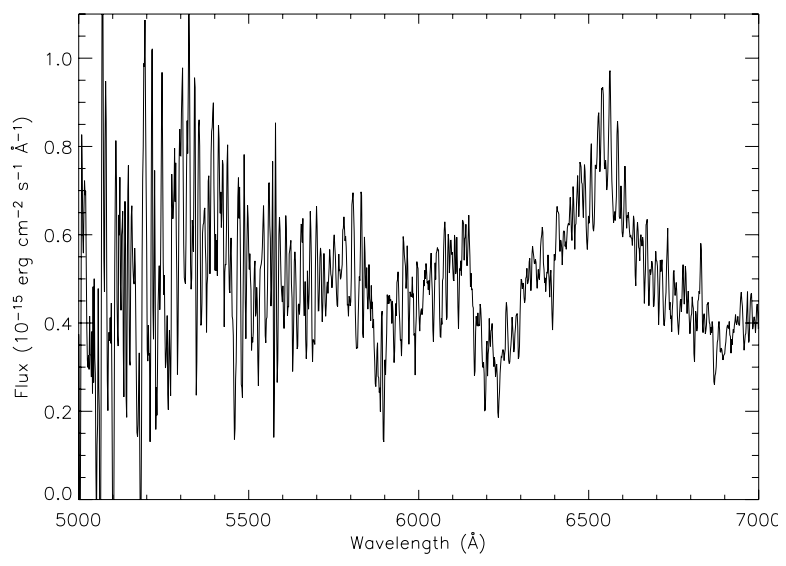

Fig. 8. Optical spectrum of RX J0507.1-6743.

count rate inferred from the first observation was a factor of $4 \pm 1$ lower. The hardness ratios HR1 and HR2 together with a blackbody spectral fit indicate a very soft source $(k T \sim 20-100 \mathrm{eV})$ with an absorption due to LMC gas and intrinsic to the source, which is considerable $\left(N_{\mathrm{H}}^{\mathrm{LMC}} \approx\right.$ a few $\left.10^{21} \mathrm{~cm}^{-2}\right)$. A galactic foreground $\mathrm{CV}$ cannot be excluded from the $\mathrm{X}$-ray data alone.

We find an optical star at a distance of 1.'9 from the X-ray position, which is most likely the optical counterpart. The optical spectrum taken at SAAO resembles the spectrum of a cool $(\$ 3500 \mathrm{~K})$ stellar atmosphere that is heavily absorbed (Lejeune et al. 1997). This is consistent with the high absorption indicated by the X-ray spectrum, which would then be caused by the stellar wind of the late type star. The expected brightness of the star is limited by the size of the Roche lobe and would be compatible with the observed brightness $(R \sim 16.5)$ for a giant star in a $\sim 370$ day orbit around a one solar mass white dwarf. Thus, the likely optical counterpart of J0507 is consistent with a M-type giant star at LMC distance, and J0507 is likely a symbiotic system in the LMC. The optical spectrum shows no strong emission lines, which could imply that the contribution of an accretion disk is minor or that the optical spectrum was taken when the (variable) X-ray source was at a lower X-ray luminosity level.

A possible periodicity of $\sim 374$ days was found in the $B$ band MACHO data of the probable optical counterpart of J0507. Although the period is close to one year, the non-detection in $R$ and the (weak) detection of a somewhat shorter period from a control star suggests that the $\sim 374$ day period is intrinsic to the source. In this case, the detection of periodicity in the blue and not in the red band data would argue against stellar pulsations of a giant star, and would favor orbital modulation, i.e. variable obscuration of the hot star due to a stellar wind from the cool star.

A high extinction is consistent with the $B-R$ color. The small optical amplitude of the modulation argues against a thermonuclear outburst cycle. The XMM-Newton observations of the X-ray bright and faint state of J0507 coincide with the blue optical maximum and minimum respectively (Fig. 5). Also, the times of the reported bright and faint X-ray states observed with ROSAT PSPC (Haberl \& Pietsch 1999b) fall in corresponding phase intervals of the optical light curve. This is consistent with viewing an optically bright accretion disk in front of and behind the optical (giant) star. Thus, it is likely that the $\sim 374$ day period is the orbital period of the J0507 binary system.

Such a large orbital period is consistent with a symbiotic system and a giant as the cool component. We note that the orbital period of the symbiotic nova RR Tel is 387 days; J0507 may 
differ from RR Tel as it is not a (recurrent) nova, but rather a persistent super-soft source (at least for the $\sim 10$ years since it was observed with ROSAT). This is consistent with the optical and infrared magnitudes that require a late K- to early M-type giant that fills the Roche lobe for such an orbital period. The optical spectrum we obtained from J0507 (Fig. 8) is consistent with such a spectral classification. Such a symbiotic system falls well into the distribution of orbital periods and Roche lobe filling of symbiotic stars predicted by the population synthesis calculations of Lü et al. (2006; see also Yungelson et al. 1995). For a system with such an orbital period, a WD with a mass of $\sim 0.5-0.8 M_{\odot}$ is predicted from the calculations of Yungelson et al. (1995). The steady nuclear burning rate of such a lower mass WD is $\sim 10^{37} \mathrm{erg} \mathrm{s}^{-1}$ and consistent with the X-ray luminosity constrained from the EPIC-pn data. Thus, J0507 would be a canonical wide-binary super-soft source.

\subsection{Upper limit count rate for XMMU J052016.0-692505}

We note that the white dwarf Be/X-ray binary candidate XMMU J052016.0-692505 (see Kahabka et al. 2006 for results from a XMM-Newton observation in January 2004) was in the field of view of another XMM-Newton observation (rev. 1210, Obs. ID = 0311591201) performed on 2006 July 18, from 14:13 to $19: 15$. The source was at a large off-axis angle of $\sim 10^{\prime}$ and had a low exposure of $\sim 5 \mathrm{ksec}$. It was not detected and we derive a $3 \sigma$ upper limit count rate $(0.2-1.0 \mathrm{keV})$ from the observed counts in the source circle of $\sim 7 \times 10^{-3} \mathrm{~s}^{-1}$. This upper limit count rate is consistent with the count rate of $2.4 \pm 0.6 \times 10^{-3} \mathrm{~s}^{-1}$ observed during an XMM-Newton observation in 2004 January (Kahabka et al. 2006).

\section{Comparison with the previously-known sample}

With the discovery of four new super-soft sources with $X M M$ Newton in the field of the LMC we increase the number of known super-soft sources (including candidates) to 18. The sample known so far comprises five close-binary, super-soft sources, one classical post-nova, a likely WD Be/X-ray binary, a widebinary (symbiotic) system, one planetary nebula, one PG1159 star, and seven transient and/or faint super-soft sources that have so far not been optically identified (cf. Table 4).

Although there is a diversity of systems, all opticallyidentified systems are consistent with a hot WD condition. Two of the systems correlate with a planetary nebula or PG1159 star, the remaining with steady or stable nuclear burning WDs.

\section{Conclusions}

We analysed archival XMM-Newton observations in the direction of the LMC and discovered four new faint super-soft sources with EPIC-pn count rates (corrected for vignetting) of $\sim(1-$ 14) $\times 10^{-3} \mathrm{~s}^{-1}$. Kahabka et al. (2006) discussed one source as a likely WD/Be X-ray binary. The likely identification of one of the new super-soft sources, J050803, with the planetary nebula LMC SMP 29 in the LMC adds one super-soft planetary nebula to the LMC sample. A second source, J052530, may be faint due to a high LMC absorbing column. It could be a close-binary, super-soft LMC source. A third source, J052215, is less strongly absorbed and may be either less luminous (if it is located in the LMC) or a Galactic foreground source. We also detected the ROSAT super-soft source J0537 with XMM-Newton. We detected the ROSAT super-soft source J0507 with XMM-Newton as an absorbed super-soft source. It is optically identified with a $M A C H O$
Table 4. Known super-soft sources in the LMC field (including candidates).

\begin{tabular}{lcc}
\hline \hline Name & Type $^{a}$ & Ref $^{b}$ \\
\hline RX J0439.8-6809 & PG1159 star & 4 \\
RX J0454.0-6900 & no ID & 7 \\
RX J0507.1-6743 & $B \sim 17.7$ & 7,3 \\
XMMU J050803.1-684017 & PN ? & 3 \\
RX J0513.9-6951 & CBSS & 1 \\
XMMU J052016.0-692505 & WD Be/XRB & 2 \\
XMMU J052215.0-701656 & no ID & 3 \\
XMMU J052530.5-671501 & $B \sim 19.2$ & 3 \\
N LMC 1995 & CV-N & 1 \\
RX J0527.8-6954 & CBSS & 1 \\
RX J0529.4-6713 & no ID & 6 \\
RX J0531.9-7038 & no ID, cand. & 7 \\
RX J0535.5-7026 & no ID, cand. & 7 \\
RX J0537.7-7034 & CBSS & 1,3 \\
CAL 83 & CBSS & 1 \\
CAL 87 & CBSS & 1 \\
RX J0549.7-7000 & no ID, cand. & 7 \\
RX J0550.9-7151 & no ID & 5 \\
\hline
\end{tabular}

${ }^{a}$ Close-binary super-soft source (CBSS), classical post-nova (CV-N), WD Be/X-ray binary (WD Be/XRB), planetary nebula (PN), no optical identification (no ID).

$b$ 1) Kahabka \& van den Heuvel (2006) and references therein, 2) Kahabka et al. (2006), 3) this paper, 4) Greiner et al. (1994), 5) Reinsch et al. (1999), 6) Kahabka (2002), 7) Haberl \& Pietsch (1999a).

variable star in the $B$-band with a period of $\sim 374$ days, which is most likely the orbital period of a symbiotic system. Thus, J0507 may be a wide-binary, super-soft source. The performed and available XMM-Newton observations of the LMC field cover only a fraction of the LMC field. All newly-discovered, supersoft X-ray sources are found in the LMC bar region.

Acknowledgements. The XMM-Newton project is supported by the Bundesministerium für Wirtschaft und Technologie/Deutsches Zentrum für Luft- und Raumfahrt (BMWI/DLR, FKZ 50 OX 0001) and the Max-Planck Society. This research has made use of the VizieR catalog access tool, CDS, Strasbourg, France. This paper utilizes public domain data originally obtained by the $M A C H O$ Project, whose work was performed under the joint auspices of the US Department of Energy, National Nuclear Security Administration by the University of California, Lawrence Livermore National Laboratory under contract No. W-7405-Eng-48, the National Science Foundation through the Center for Particle Astrophysics of the University of California under cooperative agreement AST-8809616, and the Mount Stromlo and Siding Spring Observatory, part of the Australian National University. We used the NOAO (National Optical Astronomical Observatory) IRAF (Image Reduction and Analysis Facility) software package, with Brent Miszalski's extractor task for IRAF, in conjunction with the MSSSO Karma software package and the Figaro software package from the Starlink Project. We also were granted observation time at the South African Astronomical Observatory (SAAO) and wish to thank them for their kind help and accommodations. Travel to the SAAO was funded by Australian Government Education Science and Training (DEST) - Access to Major Research Facilities Programme - No. 06/07-0-10.

\section{References}

Anders, E., \& Grevesse, N. 1989, GeCoA, 53, 197

Aschenbach, B., Briel, U. G., Haberl, F., et al. 2000, in X-ray Optics, Instruments, and Mirrors III, ed. J. E. Trümper, \& B. Aschenbach, Proc. SPIE, 4012, 731

Brüns, C., Kerp, J., Staveley-Smith, L., et al. 2005, A\&A, 432, 937

Cash, W. 1979, ApJ, 228, 939

DiStefano, R., Kong, A., \& Primini, F. A. 2006, [arXiv: astro-ph/0606364]

Greiner, J., Hasinger, G., \& Thomas, H.-C. 1994, A\&A, 281, L61

Greiner, J., Orio, M., \& Schwarz, R. 2000, A\&A, 355, 1041 
Haberl, F., \& Pietsch, W. 1999a, A\&AS, 139, 277

Haberl, F., \& Pietsch, W. 1999b, A\&A, 344, 521

Haberl, F., Dennerl, K., \& Pietsch, W. 2003, A\&A 406, 471

Kahabka, P. 2002, A\&A, 388, 100

Kahabka, P. 2006, Supersoft X-ray sources, in Adv. Space Res., 38, 2836

Kahabka, P., \& Haberl, F. 2006, A\&A, 452, 431

Kahabka, P., \& van den Heuvel, E. P. J. 1997, ARA\&A, 35, 69

Kahabka, P., \& van den Heuvel, E. P. J. 2006, in Compact Stellar X-Ray Sources,

ed. W. H. G. Lewin, \& M. van der Klis, Cambridge University Series, 461

Kahabka, P., Haberl, F., Payne, J. L., \& Filipović, M. D. 2006, A\&A, 458, 285

Leisy, P., Dennefeld, M., Allard, C., et al. 1997, A\&AS, 121, 407

Lejeune, Th., Cuisinier, F., \& Buser, R. 1997, A\&AS, 125, 229

Lomb, N. R. 1976, Ap\&SS, 39, 447

Lü, G., Yungelson, L., \& Han, Z. 2006, MNRAS, 372, 1389
Orio, M., Della Valle, M., Massone, G. \& Ögelman, H. 1997, A\&A, 325, L1

Pietsch, W., Fliri, J., Freyberg, M., et al. 2005, A\&A, 422, 879

Rappaport, S., DiStefano, R., \& Smith, D. 1994, ApJ, 426, 692

Reinsch, K., van Teeseling, A., Beuermann, K., \& Thomas, H.-C. 1999, in Highlights in X-ray Astronomy, ed. B. Aschenbach, \& M. J. Freyberg, MPE Rep. 272,70

Sasaki, M., Haberl, F., \& Pietsch, W. 2000, A\&AS, 143, 391

Scargle, J. D. 1982, ApJ, 263, 835

Šimon, V. 2003, A\&A, 406, 613

Strüder, L., Briel, U., Dennerl, K., et al. 2001, A\&A, 365, L18

Turner, M. J. L., Abbey, A., Arnaud, M., et al. 2001, A\&A, 365, L27

van den Heuvel, E. P. J., Bhattacharya, D., Nomoto, K., \& Rapppaport, S. A. 1992, A\&A, 262, 97

Yungelson, L., Livio, M., Tutukov, A., \& Kenyon, A. S. 1995, ApJ, 447, 656 